Baltic Astronomy, vol.0, 00-00, 1996.

Proc. Internat. Cooperation in Dissemination of Astronomical Data, July 3-5, 1996, St.-Petersburg, Russia

\title{
WWW ACCESS TO RADIO MEASUREMENTS OF CLUSTERS OF GALAXIES
}

\author{
A.G. Gubanov ${ }^{1}$ and H. Andernach ${ }^{2}$ \\ ${ }^{1}$ Astronomical Institute, St.-Petersburg State University, 198904 Russia \\ ${ }^{2}$ IUE Observatory, Villafranca, Apdo. 50727, E-28080 Madrid, Spain
}

Received July 20, 1996.

\begin{abstract}
Radio measurements of rich Abell clusters of galaxies have been collected both from radio source catalogues and dedicated publications. By gathering these data for every cluster, complemented by optical data, an overall picture of each cluster may be obtained and samples may be drawn suitable for statistical studies.
\end{abstract}

Key words: clusters: general; clusters: radio sources; databases

Radio sources in clusters of galaxies provide an important sample to study the physics of the intracluster medium, its influence on radio sources, and the overall evolution of extragalactic radio sources. Numerous cluster observations are available in different spectral ranges but the relevant data are spread over hundreds of publications and are heterogeneous with respect to quality, sensitivity, angular resolution, availability of an optical identification, etc.. They suffer from different selection effects, and only part of them is available in electronic form. This makes it difficult to draw correct astrophysical conclusions from a combination of different radio data.

To overcome these obstacles and to allow more efficient selection of source samples suitable to answer astrophysical questions about cluster radio emission, we are developing a database of radio sources in clusters of galaxies. It is based on the CGI interface and HTML forms and accessible on the WWW server of the Astronomical Institute of Sankt-Petersburg University (AISPbU) under URL

http://WWW.aispbu.spb.su/WWW/Clusters.html 
The individual source measurements were extracted partly from the Radio Astronomical Catalogues (RAC) database of AISPbU (Gubanov \& Titov 1996) and partly from many publications dedicated to cluster radio emission. Dozens of published source tables were converted by us into electronic form for the first time, using a page scanner with "Optical Character Recognition" software (OCR) and special proof-reading tools (cf. Andernach et al. 1996, these proceedings).

We currently include in our service radio and optical data for all 4076 rich clusters in the ACO catalogue (Abell et al. 1989). Inclusion of X-ray information and properties of the brightest cluster galaxies is planned. We try to realise the following basic ideas in this service:

- gather in one place all major information for a given cluster;

- create an overall picture of the cluster based on measurements;

- give a clear graphic presentation of different cluster data;

- allow users to provide corrections and supply new data;

- provide tools for statistical work with cluster data.

At present we have built the necessary tools for all except the last item. The URL given above allows to extract and display either radio or optical data for a user-specified cluster.

The page with radio data begins with comments on certain radio sources, e.g. on cluster membership of sources, ambiguities of the interpretation of data, etc. This is followed by a section on individual radio galaxies in the cluster, including the optical identification and radio spectral data, sorted by observing frequency and converted to a common absolute flux scale where possible. The last block of the page gives the data as published from all relevant references for radio sources within a projected distance of one Abell radius from their centres $\left(\mathrm{R}_{\mathrm{a}}=3 \mathrm{Mpc}\right.$ for $\mathrm{H}_{\circ}=50$ $\left.\mathrm{kms}^{-1} \mathrm{Mpc}^{-1}\right)$. More detail on these references is available and in future we plan to provide hyperlinks to abstract services or to electronic journals. Further links allow to extract entries from the preliminary source list of the NRAO VLA Sky Survey (NVSS, Condon et al. 1996) and to display the Digitized Sky Survey image of a $500 \times 500 \mathrm{kpc}^{2}$ region around a given cluster radio galaxy.

The page with optical data lists the basic optical parameters of the cluster, as well as published notes and relevant references. We began to provide links to optical object catalogs from APM (Irwin et al. 1994) which may be displayed as PostScript finding charts, currently limited to a maximum field of $30^{\prime} \times 30^{\prime}$, i.e. less than $1 \mathrm{R}_{\mathrm{a}}$ for nearby clusters. A more promising tool are Tcl applets, which permit to run application programs on the user's local computer within the WWW connection. Applets allow to show the relevant parameters of the displayed objects simply by posi- 
tioning the cursor on them. They require less disk space than PostScript and allow the user to control the design of the charts. We plan to use Tcl applets later to link the optical images of objects in the cluster with any relevant information available on them, like radio parameters, spectra, redshifts, etc. We currently use Tcl applets either with the SurfIt! browser (Titov 1996, these proceedings) or with the netscape browser using the "Simple windowing shell" (wish) program. These should also directly work under the netscape browser soon.

We anticipate that many users are more familiar with specific sections of data than we are. To improve the quality and completeness of the cluster database it is thus important that the user may provide corrections or additions to the data in an as comfortable fashion as possible. Rather than having to compose a separate email message, the user may simply prepare revised versions of the data "on the fly" without leaving their browsers, using xedit (under X-windows) or the vi editor (otherwise). Revisions are folded into the database upon our check and approval. Depending on first experience with input from users we can arrange for other editors if needed.

The database is being updated permanently with new cluster radio and optical measurements, e.g. from the NRAO VLA Sky Surveys NVSS and FIRST (Becker et al. 1995), or with optical objects from APM, COSMOS (Drinkwater et al. 1995) and APS (cf. Odewahn 1995).

Our service is intended as an open and collaborative enterprise to the benefit of cluster research. We welcome users to join our efforts, to tell us their preferences for data to be included and to contribute new data sets, especially those not previously available in electronic form.

This work is supported by the RFBR grant N 94-07-20441.

\section{REFERENCES}

Abell G.O., Corwin Jr. H.G. \& Olowin R.P. 1989, ApJS 70, 1

Becker R.H., White R.L. \& Helfand D.J. 1995, ApJ, 450, 559

Condon J.J. et al. 1996, AJ, to be submitted

Drinkwater M.J., Barnes D.G. \& Ellison S.L. 1995, Proc. ASA, 12, 248

Gubanov A.G. \& Titov V.B. 1996, Vestnik St.-Petersburg Univ., Ser. 1, Vyp. 3 (N 15), in press

Irwin M., Maddox S., \& McMahon R. 1994, Spectrum, Royal Obs. 2, 14

Odewahn S. C. 1995, PASP 107, 770 